\title{
TheWhite Blood Cell Count to Hemoglobin Level Ratio is Correlated with the Presence of Cortical Defects on DMSA Renal Scans in Children with Febrile Urinary Tract Infection
}

\author{
Bo Kyeong Jin, M.D. \\ Kyung Suk Baek, M.D. \\ Seon Kyeong Rhie, M.D. \\ Jun Ho Lee, M.D., Ph.D. \\ Department of Pediatrics CHA Bundang \\ Medical Center, CHA University School \\ of Medicine, Seongnam, Korea \\ Corresponding author: \\ Jun Ho Lee, MD, PhD, \\ Department of Pediatrics, CHA Bundang \\ Medical Center, CHA University School \\ of Medicine, 59, Yatap-ro, Bundang-gu, \\ Seongnam-si, Gyeonggi-do, 13496, Korea \\ Tel: $+82-31-780-5011$ \\ Fax:+82-31-780-5011 \\ E-mail: naesusana@gmail.com \\ Corresponding author: \\ Seon Kyeong Rhie, M.D. \\ Department of Pediatrics, CHA Bundang \\ Medical Center, CHA University School \\ of Medicine, 59, Yatap-ro, Bundang-gu, \\ Seongnam-si, Gyeonggi-do, 13496, Korea \\ Tel: +82-31-780-5011 \\ Fax: $+82-31-780-5011$ \\ E-mail: starclusters@gmail.com \\ Received: 16 July 2018 \\ Revised: 20 September 2018 \\ Accepted: 28 September 2018
}

This is an open-access article distributed under the terms of the Creative Commons Attribution Non-Commercial License (http:// creativecommons.org/licenses/by-nc/4.0/) which permits unrestricted non-commercial use, distribution, and reproduction in any medium, provided the original work is properly cited.

Copyright (C) 2018The Korean Society of Pediatric Nephrology
Purpose: We investigated whether the white blood cell (WBC) count to hemoglobin ( $\mathrm{Hgb}$ ) level ratio is correlated with the presence of cortical defects on dimercaptosuccinic acid (DMSA) renal scan in children with febrile urinary tract infection (UTI).

Methods: We examined 95 children who were consecutively admitted to our hospital with their first episode of febrile UTI. Blood tests (C-reactive protein [CRP], WBC, Hgb] were performed. All enrolled children underwent DMSA scanning during admission. Data were compared between children with positive and negative DMSA results. The correlations between WBC to $\mathrm{Hgb}$ ratio and the presence of cortical defects on DMSA scan, and between WBC to Hgb ratio and CRP level were analyzed using the Pearson chi-squared test. Multiple logistic regression analysis was used to evaluate whether WBC to $\mathrm{Hgb}$ ratio could predict the cortical defects on DMSA scan in children with febrile UTI.

Results: The WBC to Hgb ratio was significantly higher in children with positive DMSA results than in those with negative DMSA results; positively correlated with the presence of cortical defects on DMSA scan and CRP; and was a significant factor for predicting the presence of cortical defects on DMSA scan.

Conclusion: The WBC to Hgb ratio may predict the presence of cortical defects on acute DMSA scans in children with febrile UTI.

Key words: Hemoglobin, Pyelonephritis, Urinary tract infection, White blood cell count

\section{Introduction}

Febrile urinary tract infection (UTI) is common in children, especially those under two years old who are immunocompromised hosts or have severe congenital urinary tract anomalies. Febrile UTI is considered as upper UTI or pyelonephritis. However, according to data from acute dimercaptosuccinic acid (DMSA) scans routinely performed in children with febrile UTI regardless of how urine samples were collected before the year of 2011, only less than $30 \%$ of them had positive DMSA renal scan results ${ }^{11}$.

Children with febrile UTI but with negative DMSA results have pyelitis or lower UTI with other fever focus and manifesting only fever initially. Only pyelonephritis can produce acquired renal scarring, and only recurrent pyelonephritis can lead to chronic renal insufficiency. Therefore, among children 
with febrile UTI, determining the presence of pyelonephritis is very important. However, positive urine culture results, which is currently the gold standard in the diagnosis of acute pyelonephritis, cannot predict the presence of cortical defects on DMSA scan in children with febrile UTI, although urine sampling was appropriately collected. Furthermore, urine culture results cannot contribute to making treatment decisions during clinical visits. Nevertheless, performing DMSA renal scans in all children with febrile UTI is not acceptable because of its invasiveness.

Due to these reasons, trying to find simple, cheap, prompt, and more accurate diagnostic methods than serum C-reactive protein (CRP), white blood cell (WBC) count, or urine culture for determining the presence of pyelonephritis among children with febrile UTI is challenging to pediatricians.

Authors previously reported that mean hemoglobin (Hgb) level was significantly lowered in children with positive DMSA results than in those with negative DMSA results ${ }^{2}$.

In the present study, we investigated whether WBC to $\mathrm{Hgb}$ ratio could be helpful for determining the presence of cortical defects on DMSA scan in children with febrile UTI.

\section{Materials and methods}

Among 124 consecutive children admitted to our hospital with their first episode of febrile UTI from July 2013 to June 2015, 95 children with completed data sheets were included in this study. Twenty-two children who did not undergo a DMSA renal scan at the time of acute infection and seven children with missing data were excluded. Febrile UTI was defined as follows: high fever $\geq 38^{\circ} \mathrm{C}$, abnormal urinalysis (pyuria [ $>5 \mathrm{WBC}$ counts/high-power field] and positive leukocyte esterase results), positive CRP results (>0.3 mg/dL), and lack of other fever focus. Urine was sampled by collecting a midstream urine specimen in toddlers or in older children and using the clean catch bag method in non-toilet trained children. Significant bacteriuria (BU) was defined as the presence of $\geq 10^{5}$ colonyforming units/high-power field of a single-strain isolate. All the enrolled children with febrile UTI underwent a DMSA renal scan upon admission. In addition, blood (Creactive protein $[\mathrm{CRP}]$, white blood cell $[\mathrm{WBC}]$ count, he- moglobin $[\mathrm{Hgb}])$ tests were performed at their admission.

DMSA scans were performed using the planar technique with a dual-headed Symbia E gamma camera (Siemens, Erlangen, Germany) equipped with low-energy high-resolution collimator ( $140 \mathrm{KeV}, 20 \%$ symmetric window) and were interpreted by two nuclear medicine consultants. A positive DMSA result (indicative of cortical defects) was defined as reduced or absent tracer localization and indistinct margins that did not deform the renal contour.

Written informed consent was obtained from the parents of all febrile UTI children prior to DMSA scans. The CHA University Institutional Review Board approved this study, including the consent procedure (CHA IRB No.2018-06057).

All data were collected retrospectively and were compared between the children with the positive and negative DMSA results. The correlation was analyzed between WBC to Hgb ratio and the presence of cortical defects on DMSA scan and between WBC to Hgb ratio and CRP.

\section{Statistical analysis}

All variables are presented as mean \pm standard deviation, and continuous variables were analyzed using the Student's t-test. Qualitative variables and correlations were analyzed using the Pearson chi-squared test and Pearson correlation coefficient (two-tailed probability), respectively. Non-parametric variables were analyzed using Spearman's rank correlation coefficient. Using multiple logistic regression analysis (MLRA), the ability of WBC to Hgb ratio to predict the presence of cortical defects on DMSA scan was investigated. The areas under the receiver operating characteristic (ROC) curves of relevant factors and the associated levels of significance were evaluated when the value of the state variable was 1 . Statistical analysis was performed using SPSS statistics 22 (SPSS Inc, Chicago, IL). Statistical significance was defined as $P \leq 0.05$.

\section{Results}

\section{Demographic and laboratory findings of the} study population

Nine children (20\%) with positive DMSA results were diagnosed with culture-negative pyelonephritis. There were 
no differences in congenital anomalies, including hydronephrosis, on renal sonogram between children with positive and negative DMSA results. Congenital hypoplastic kidney (relative renal uptake of less than 35\%) was not detected on DMSA scan in this study. Voiding cystourethrogram was not performed at the first episode of acute pyelonephritis.

The study group included children aged 0 to 109 months, with a mean age of 10.8 months. There was no difference in the age and sex ratio between the two groups. CRP, WBC count, $\mathrm{WBC} / \mathrm{Hgb}$ ratio were significantly higher in children with positive DMSA results than in those with negative DMSA results (7.4 44.1 vs. $2.7 \pm 2.7, P<0.001 ; 18,771 \pm 5,817$ vs. $13,033 \pm 6,241, P<0.001 ; 1,721 \pm 579$ vs. $1,138 \pm 456, P<0.001$, respectively). Hgb levels were significantly lower in children with positive DMSA results than in those with negative DMSA results $(10.6 \pm 0.69$ vs. $11.4 \pm 0.89, P=0.005)$ (Table 1 ).

\section{Significant correlations between parameters in the study population}

The presence of cortical defects on acute DMSA scan correlated positively with $\mathrm{CRP}, \mathrm{WBC}$, and $\mathrm{WBC} / \mathrm{Hgb}$ ratio and negatively with $\mathrm{Hgb}(\mathrm{r}=0.55436, P<0.001 ; \mathrm{r}=0.49031$, $P<0.001 ; \mathrm{r}=0.49031, P<0.001 ; \mathrm{r}=-0.26319, P=0.01$, respectively). CRP correlated positively with $\mathrm{WBC}$ and $\mathrm{WBC} / \mathrm{Hgb}$ ratio and negatively with $\mathrm{Hgb}(\mathrm{r}=0.40457, P<0.001 ; \mathrm{r}=$ $0.37390, P<0.001 ; \mathrm{r}=-0.30751, P=0.002$, respectively). WBC count was not correlated with $\mathrm{Hgb}(\mathrm{r}=-0.16374, P=0.11)$.

Table 1. Comparison of the Mean Laboratory Data for Children with Positive and Negative DMSA Results

\begin{tabular}{lccr}
\hline & DMSA (+) & DMSA (-) & $P$ value \\
\hline N & 45 & 50 & \\
Age (months) & $10.1 \pm 16.5$ & $11.5 \pm 13.8$ & 0.57 \\
M:F & $29: 16$ & $34: 16$ & \\
CRP $(\mathrm{mg} / \mathrm{dL})$ & $7.4 \pm 4.1$ & $2.7 \pm 2.7$ & $<0.001^{*}$ \\
WBC $\left(/ \mathrm{mm}^{3}\right)$ & $18,771 \pm 5,817$ & $13,033 \pm 6,241$ & $<0.001^{*}$ \\
Hemoglobin (Hgb, g/dL) & $10.6 \pm 0.69$ & $11.4 \pm 0.89$ & $0.005^{*}$ \\
WBC/Hgb ratio & $1,721 \pm 579$ & $1,138 \pm 456$ & $<0.001^{*}$ \\
\hline
\end{tabular}

The laboratory examination results are expressed as mean \pm standard deviation.

Abbreviations: DMSA, Technetium 99m-dimercaptosuccinic acid; N, number of patients; M, male; F, female; CRP, C-reactive protein; WBC, white blood cell count

$* P<0.05$.

\section{MLRA of WBC/Hgb ratio to predict the presence of} cortical defects on acute DMSA scan

Using MLRA, Table 2 demonstrates that $\mathrm{WBC} / \mathrm{Hgb}$ ratio was a significant factor for predicting the presence of cortical defects in acute DMSA scan similar to CRP (Table 2).

\section{ROC curves and $95 \%$ confidence interval ( $\mathrm{Cl})$ of CRP, WBC, WBC/Hgb ratio and $\mathrm{Hgb}$ to predict the presence of cortical defects on acute DMSA scan}

The areas of the ROC curves and 95\% CI of CRP, WBC, $\mathrm{WBC} / \mathrm{Hgb}$ ratio and $\mathrm{Hgb}$ to predict the presence of cortical defects on acute DMSA scan were significant (Table 3).

\section{Discussion}

There are many possible advantages if the exact prediction of cortical defects through acute DMSA scans in children with febrile UTI. First, admission is not necessary in children with pyelitis or lower UTI with other fever foci even if they are less than 12 months old. Second, performing imaging studies according to UTI guidelines is not necessary if UTI is not recurrent. To some extent, the possibility of recurrent febrile UTI or developing acquired renal scars in the future can be individually predicted. Total medical expenses at the national level can be reduced.

There are disadvantages in urine culture results being considered as a gold standard for a diagnosis of pyelonephritis: if all children with positive urine culture results among children with febrile UTI might have cortical defects on acute DMSA scans, which might result in false po-

Table 2. Results of Multiple Logistic Regression Analyses for C- reactive Protein and White Blood Cell to Hemoglobin Ratio to Predict the Presence of Cortical Defects on Acute DMSA Scanning [DMSA $(+)]$.

\begin{tabular}{lccc}
\hline DMSA (+) & $P$-value & OR & $95 \% \mathrm{Cl}$ \\
\hline CRP & $<0.000^{*}$ & 1.368 & $1.164-1.607$ \\
WBC & $0.045^{*}$ & 1.009 & $1.000-1.018$ \\
Hgb & 0.529 & 0.836 & $0.479-1.460$ \\
\hline DMSA (+) & $P$-value & OR & $95 \% \mathrm{Cl}$ \\
\hline CRP & $<0.001^{*}$ & 1.360 & $1.153-1.604$ \\
WBC/Hgb & $0.004^{*}$ & 1.002 & $1.001-1.003$ \\
\hline
\end{tabular}

Abbreviations: DMSA, 99m-dimercaptosuccinic acid; OR, odds ratio; $\mathrm{Cl}$, 95\% confidence interval; CRP, C-reactive protein; WBC, white blood cell count; Hgb, hemoglobin; WBC/Hgb, WBC to Hgb ratio. ${ }^{*} P<0.05$. 
sitive findings of urine culture are high (71\%) even though urine sampling is appropriately collected ${ }^{3)}$. Most of them include children with pyelitis or lower UTI associated with other fever foci. False negative results (15\%) of urine culture are not low even though urine sampling is appropriately collected $^{4}$. The discrimination of children with pyelonephritis from children with lower UTI and other fever foci accompanying asymptomatic bacteriuria is impossible. Urine culture is not helpful when a prompt diagnosis of pyelonephritis is needed at clinics.

Therefore, many clinicians depend on other helpful parameters correlated with pyelonephritis, such as serum $\mathrm{CRP}, \mathrm{WBC}$ or procalcitonin etc. when pyelonephritis is considered as a diagnosis in children with abnormal urinalysis. Serum WBC level is a known factor helpful for predicting the presence of cortical defects on acute DMSA scans $^{4,5)}$. Authors reported that Hgb level was significantly lower in children with positive DMSA results than in those with negative DMSA results ${ }^{2}$. Thus, authors hypothesized

Table 3. Area Under the Receiver Operating Characteristic (ROC) Curves of Relevant Factors to Predict the Presence of Cortical defects on DMSA Scanning and their associated Levels of Significance

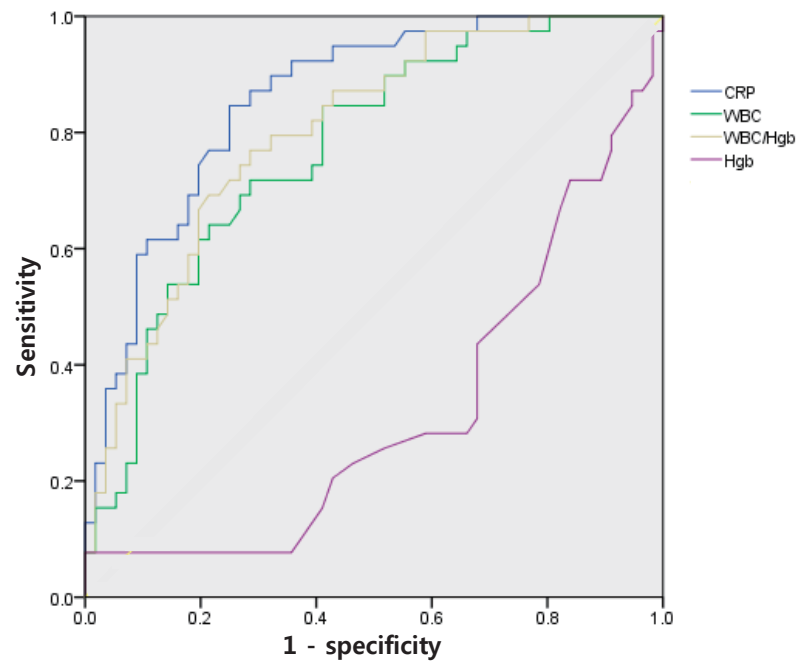

\begin{tabular}{llcc}
\hline APN & AUC & $P$-value & $95 \% \mathrm{Cl}$ \\
\hline CRP & 0.857 & $<0.001^{*}$ & $0.783-0.931$ \\
WBC & 0.773 & $<0.001^{*}$ & $0.679-0.866$ \\
$\mathrm{Hgb}$ & 0.318 & $0.003^{*}$ & $0.207-0.429$ \\
WBC/Hgb & 0.802 & $<0.001^{*}$ & $0.714-0.889$ \\
\hline
\end{tabular}

Abbreviations: AUC, areas under the ROC curve; $\mathrm{Cl}, 95 \%$ confidence interval; CRP, C-reactive protein; WBC, white blood cell count; Hgb, hemoglobin; WBC/Hgb, WBC to Hgb ratio. ${ }^{*} P<0.05$. that the combination of these two factors could be more helpful for predicting positive DMSA results in children with febrile UTI. If the cut-off value of WBC count would be $14,000 / \mathrm{mm}^{3}$, sensitivity, specificity, positive predictive value, and negative predictive value of WBC count for predicting positive DMSA results would be $80.9 \%$ [95\% CI (confidence interval), 66.7-90.9\%], 56.1\% (95\% CI, 43.3$68.3 \%$ ), 56.7\% (95\% CI, 49.1-64.0\%) and 80.4\% (95\% CI, 68.8-88.5\%). If the cut-off value of $\mathrm{WBC} / \mathrm{Hgb}$ ratio be 1200 , sensitivity, specificity, positive predictive value, and negative predictive value of $\mathrm{WBC} / \mathrm{Hgb}$ ratio for predicting positive DMSA results would be $84.6 \%$ [95\% CI, 69.5-94.1\%], 57.1\% (95\% CI, 43.2-70.3\%), 57.79\% (95\% CI, 49.7-65.7\%) and $84.2 \%$ (95\% CI, 71.2-92.0\%). The sensitivity and negative predictive value of $\mathrm{WBC} / \mathrm{Hgb}$ ratio is slightly increased compared to that of WBC count alone. Although the predicting power of $\mathrm{WBC} / \mathrm{Hbg}$ ratio is not comparable to that of serum CRP, it can be used as a complementary tool for evaluation.

The reason why Hgb level decreases in children with positive DMSA results is unknown. However, authors thought that their decreased Hgb level could be due to dilutional effect resulting from the activation of intrarenal renin-angiotensin-aldosterone system in the pathogenesis of acute pyelonephritis ${ }^{2)}$.

The limitations of this study are as follows: $\mathrm{WBC} / \mathrm{Hgb}$ ratio is not better compared to other factors by a large margin. Other factors influencing Hgb levels like nutrition, nutritional supplements, socioeconomic status, or previous Hgb levels were not considered. The reason for decreased $\mathrm{Hgb}$ level in children with the positive DMSA results is currently unclear. Follow-up of their Hgb levels is needed.

In conclusion, $\mathrm{WBC} / \mathrm{Hgb}$ ratio may help predict the presence of cortical defects on acute DMSA scans in children with febrile UTI.

\section{Patient consent}

This study was approved by the institutional review board (IRB), and the consent was waived due to the nature of the retrospective study [CHA IRB No.2018-06-057]. 


\section{Conflicts of interest}

No potential conflict of interest relevant to this article was reported.

\section{References}

1. Lee JH, Kim MK, Park SE. Is a routine voiding cystourethrogram necessary in children after the first febrile urinary tract infection? Acta Paediatr 2012;101:e105-9.

2. Jung SJ, Lee JH. Hemoglobin level is a more predictive diagnostic marker for acute pyelonephritis than mean platelet volume. Clin
Exp Nephrol 2016;20:823-4.

3. Supavekin S, Srapaitoolkorn W, Pravisithikul N, Kutanavanishapong S, Chiewvit S. The role of DMSA renal scintigraphy in the first episode of urinary tract infection in childhood. Ann Nucl Med 2013;27:170-6.

4. Lee JH. Discrimination of culture negative pyelonephritis in children with suspected febrile urinary tract infection and negative urine culture results. J Microbiol Immunol Infect 2017 Oct 25. Pii: S1684-1182(17)30233-5. Doi: 10.1016/j.jmii.2017.09.005. [Epub ahead of print].

5. Ansari Gilani K, Modaresi Esfeh J, Gholamrezanezhad A, Gholami A, Mamishi S, Eftekhari M etc. Predictors of abnormal renal cortical scintigraphy in children with first urinary tract infection: the importance of time factor. Int Urol Nephrol 2010;42:1041-7. 